\title{
The Onset of Ferromagnetic Convection in a Micropolar Ferromagnetic Fluid Layer Heated from Below
}

\author{
C. E. Nanjundappa ${ }^{1}$, I. S. Shivakumara ${ }^{2}$, K. Srikumar ${ }^{3}$ \\ ${ }^{1}$ Department of Mathematics, Ambedkar Institute of Technology, Bangalore, India; ${ }^{2}$ UGC-CAS in Fluid Mechanics, Department of \\ Mathematics, Bangalore University, Bangalore, India; ${ }^{3}$ Department of Mathematics, East Point College of Engineering for Women, \\ Bangalore, India. \\ Email: cenanju@hotmail.com
}

Received May $13^{\text {th }}, 2012$; revised June $20^{\text {th }}, 2012$; accepted July $10^{\text {th }}, 2012$

\begin{abstract}
The onset of ferromagnetic convection in a micropolar ferromagnetic fluid layer heated from below in the presence of a uniform applied vertical magnetic field has been investigated. The rigid-isothermal boundaries of the fluid layer are considered to be either paramagnetic or ferromagnetic and the eigenvalue problem is solved numerically using the Galerkin method. It is noted that the paramagnetic boundaries with large magnetic susceptibility $\chi$ delays the onset of ferromagnetic convection the most when compared to very low magnetic susceptibility as well as ferromagnetic boundaries. Increase in the value of magnetic parameter $M_{1}$ and spin diffusion (couple stress) parameter $N_{3}$ is to hasten, while increase in the value of coupling parameter $N_{1}$ and micropolar heat conduction parameter $N_{5}$ is to delay the onset of ferromagnetic convection. Further, increase in the value of $M_{1}, N_{1}, N_{5}$ and $\chi$ as well as decrease in $N_{3}$ is to diminish the size of convection cells.
\end{abstract}

Keywords: Micropolar Ferrofluid; Ferromagnetic Convection; Paramagnetic Boundaries; Rigid Boundaries; Magnetic Susceptibility

\section{Introduction}

Ferrofluids or magnetic fluids are commercially manufactured colloidal liquids usually formed by suspending mono domain nanoparticles (their diameter is typically 3 - $10 \mathrm{~nm}$ ) of magnetite in non-conducting liquids like heptane, kerosene, water etc. and they are also called magnetic nanofluids. These fluids get magnetized in the presence of an external magnetic field and due to their both liquid and magnetic properties they have emerged as reliable materials capable of solving complex engineering problems. An extensive literature pertaining to this field and also the important applications of these fluids to many practical problems can be found in the books by Rosensweig [1], Berkovsky et al. [2] and Hergt et al. [3]. It is also recognized that these fluids have promising potential for heat transfer applications in electronics, micro and nanoelectromechanical systems (MEMS and NEMS), and air-conditioning and ventilation

Several theories were used to describe the motion of ferrofluids and amongst them the continuum description of the ferrofluids has been in existence since the work of Neuringer and Rosensweig [4]. Their theory is called "quasi-stationary theory". Based on this theory, several studies on convective instability in a ferrofluid layer have been undertaken in the past. Finlayson [5] has studied the convective instability of a magnetic fluid layer heated from below in the presence of a uniform vertical magnetic field. Gotoh and Yamada [6] have carried out the same study by assuming the fluid to be confined between two magnetic pole pieces. Stiles et al. [7] have analyzed linear and weakly nonlinear thermoconvective instability in a thin layer of ferrofluid subject to a weak uniform external magnetic field in the vertical direction. Blennerhassett et al. [8] have analyzed the heat transfer characteristics in a strongly magnetized ferrofluids. The nonlinear stability analysis for a magnetized ferrofluid layer heated from below has been performed by Sunil and Mahajan [9] for the case of stress free boundaries. Whereas, Nanjundappa and Shivakumara [10] have investigated the effects of variety of velocity and temperature boundary conditions on the onset of thermomagnetic convection in an initially quiescent ferrofluid layer in the presence of a uniform magnetic field. By using quasistationary theory but treating the ferrofluids as binary mixtures, Shliomis [11] and Shliomis and Smorodin [12] have studied convective instability of magnetized ferrofluids by considering the influence of concentration gradients and Soret effects. The latter authors have also pre- 
dicted oscillatory instability in a certain region of magnetic field and the fluid temperature. In a review article, Odenbach [13] has focused on recent developments in the field of rheological investigations of ferrofluids and their importance for the general treatment of ferrofluids.

The development of different kinds of ferrofluids exhibiting significant changes in their microstructure has outlined the need of new description for ferrofluids. It is believed that quasi-stationary theory is reasonably valid for colloidal suspensions of Néel particles in which the particle magnetic moment $\boldsymbol{m}$ rotates inside the particle and the particle does not rotate itself and hence no momentum transfer, from the particle to the fluid, occurs when the applied magnetic field has a changing direction or magnitude. On the other hand, for Brownian particle in which the vector $\boldsymbol{m}$ is locked into the crystal axis of the particle and rotates along with the particle rotation, with finite magnetic relaxation time, one has to incorporate the intrinsic rotation of the particle and there is thus momentum transfer to the carrier fluid in the form of a viscous friction. Based on these facts, the equations involving rotational or vortex viscosity and the nonequilibrium magnetization equation, involving Brownian relaxation time, are used to discuss thermoconvective instability of a ferrofluid in a strong external magnetic field by Stiles and Kagan [14]. However, more appropriate equations which allow proper consideration of internal rotation and vortex viscosity have been considered by Kaloni and Lou [15] to investigate convective instability problem in the horizontal layer of a magnetic fluid with Brownian relaxation mechanism. Recently, Paras Ram and Kushal Sharma [16] have studied the effect of magnetic field-dependent viscosity (MFD) along with porosity on the revolving Axi-symmetric steady ferrofluid flow with rotating disk.

Since the ferrofluids are colloidal suspensions of nanoparticles, as suggested by Rosensweig [1] in his monograph, it is pertinent to consider the effect of microrotation of the particles in the study. Based on this fact, studies have been undertaken by treating ferrofluids as micropolar fluids and the theory of micropolar fluid proposed by Eringen [17] has been used in investigating the problems. Micropolar fluids have been receiving a great deal of interest and research focus due to their applications like solidification of liquid crystals, the extrusion of polymer fluids, cooling of a metallic plate in a bath colloidal suspension solutions and exotic lubricants. In the uniform magnetic field, the magnetization characteristic depends on particle spin but does not on fluid velocity: Hence micropolar ferrofluid stability studies have become an important field of research these days. Although convective instability problems in a micropolar fluid layer subject to various effects have been studied extensively, the works pertaining to micropolar ferrofluids is in much-to-be desired state. Many researchers [18-23] have been rigorously investigated the Rayleigh-Benard situation in Eringen's micropolar non-magnetic fluids. From all these studies, they mainly found that stationary convection is the preferred mode for heating from below. Sharma and Kumar [24] and Sharma and Gupta [25] also gave a good understanding of thermal convection of micropolar fluids. Zahn and Greer [26] have considered interesting possibilities in a planar micropolar ferromagnetic fluid flow with an AC magnetic field. They have examined a simpler case where the applied magnetic fields along and transverse to the duct axis are spatially uniform and varying sinusoidally with time. Abraham [27] has investigated the problem of Rayleigh-Benard convection in a micropolar ferromagnetic fluid layer permeated by a uniform magnetic field for stress-free boundaries. Reena and Rana [28,29] have studied the some convection problems on micropolar fluids saturating a porous medium. Recently, Thermal instability problem in a rotating micropolar ferrofluid has also been considered by Qin and Kaloni [30] and Sunil et al. [31], and references therein.

However, the increased importance of ferrofluids in many heat transfer applications demand the study of the onset of ferromagnetic convection in a layer of micropolar ferrofluid for more realistic velocity and magnetic boundary conditions. The aim of the present paper is, therefore, to investigate the onset of ferromagnetic convection in a micropolar ferrofluid layer heated from below in the presence of a uniform vertical magnetic field by considering the bounding surfaces as rigid-isothermal and which are either paramagnetic or ferromagnetic. The resulting eigenvalue problem is solved numerically using the Galerkin technique. The critical thermal Rayleigh number and associated wave number account for the stability character.

\section{Mathematical Formulation}

The physical configuration considered is as shown in Figure 1. We consider an initially quiescent horizontal incompressible micropolar ferrofluid layer of characteristic thickness $d$ in the presence of an applied uniform magnetic field $H_{0}$ in the vertical direction with the angular momentum $\boldsymbol{\omega}$. The lower and the upper boundaries are rigid-isothermal which are either paramagnetic or ferromagnetic. Let $T_{0}$ and $T_{1}\left(<T_{0}\right)$ be the temperatures of the lower and upper rigid boundaries, respectively with $\Delta T\left(=T_{0}-T_{1}\right)$ being the temperature difference. A Cartesian co-ordinate system $(x, y, z)$ is used with the origin at the bottom of the layer and $z$-axis is directed vertically upward. Gravity acts in the negative $z$-direction, $\boldsymbol{g}=-g \hat{k}$ where $\hat{k}$ is the unit vector in the $z$-direction. 

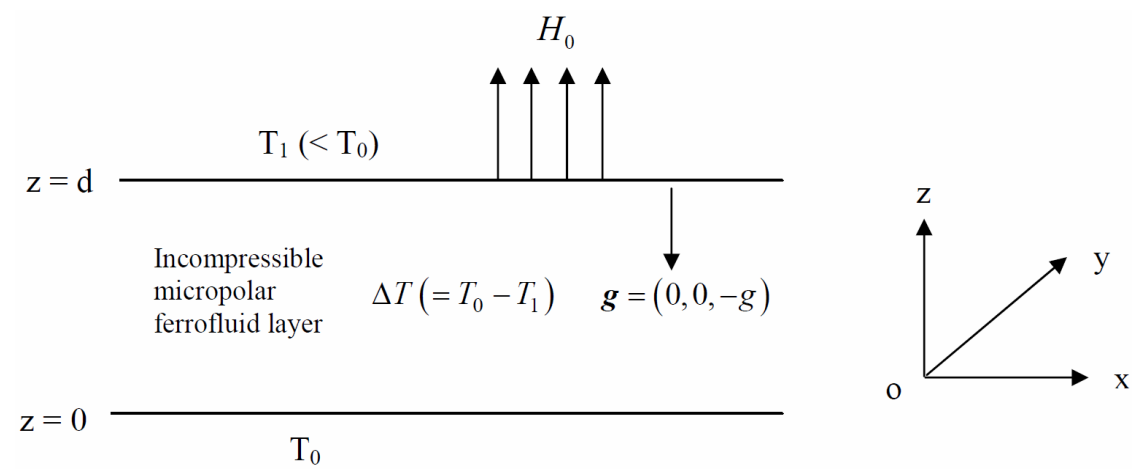

Figure 1. Physical configuration.

The basic equations governing the motion of an incompressible Boussinesq micropolar ferromagnetic fluid for the above model are as follows $[1,6,17,30]$ :

Continuity equation

$$
\nabla \cdot \boldsymbol{q}=0
$$

Angular momentum equation

$$
\begin{aligned}
\rho_{0}\left[\frac{\partial \boldsymbol{q}}{\partial t}+(\boldsymbol{q} \cdot \nabla) \boldsymbol{q}\right] & =-\nabla p+\rho \boldsymbol{g}+(\boldsymbol{B} \cdot \nabla) \boldsymbol{H} \\
& +\left(\eta+\xi_{r}\right) \nabla^{2} \boldsymbol{q}+2 \xi_{r}(\nabla \times \boldsymbol{\omega}) .
\end{aligned}
$$

Internal angular momentum equation

$$
\begin{aligned}
\rho_{0} I\left[\frac{\partial \boldsymbol{\omega}}{\partial t}+(\boldsymbol{q} \cdot \nabla) \boldsymbol{\omega}\right] & =2 \xi_{r}[(\nabla \times \boldsymbol{q})-2 \boldsymbol{\omega}]+\mu_{0}(\boldsymbol{M} \times \boldsymbol{H}) \\
& +\nabla(\nabla \cdot \boldsymbol{\omega})+\eta^{\prime}\left(\nabla^{2} \boldsymbol{\omega}\right) .
\end{aligned}
$$

Energy equation

$$
\begin{aligned}
& {\left[\rho_{0} C_{V, H}-\mu_{0} \boldsymbol{H} \cdot\left(\frac{\partial \boldsymbol{M}}{\partial T}\right)_{V, H}\right] \frac{D T}{D t}+\mu_{0} T\left(\frac{\partial \boldsymbol{M}}{\partial T}\right)_{V, H} \cdot \frac{D \boldsymbol{H}}{D t}} \\
& =k_{1} \nabla^{2} T+\delta(\nabla \times \boldsymbol{\omega}) \cdot \nabla T .
\end{aligned}
$$

Equation of state

$$
\rho=\rho_{0}\left[1-\alpha\left(T-T_{0}\right)\right] .
$$

Maxwell's equation in the magnetostatic limit

$$
\begin{gathered}
\nabla \cdot \boldsymbol{B}=0, \quad \nabla \times \boldsymbol{H}=0 \quad \text { or } \boldsymbol{H}=\nabla \phi \\
\boldsymbol{B}=\mu_{0}(\boldsymbol{M}+\boldsymbol{H}) .
\end{gathered}
$$

It is considered that the magnetization is aligned with the magnetic field and is taken as a function of both magnetic field and temperature in the form

$$
\boldsymbol{M}=\frac{\boldsymbol{H}}{H} M(H, T) .
$$

The magnetic equation of state is given by

$$
M=M_{0}+\chi\left(H-H_{0}\right)-K\left(T-T_{0}\right) .
$$

In the above equations, $\boldsymbol{q}=(u, v, w)$ is the velocity of the fluid, $p$ the pressure, $\rho$ the density, $\eta$ the shear kinematic viscosity co-efficient, $\xi_{r}$ the vortex (rotational) viscosity, $\boldsymbol{\omega}=\left(\Omega_{1}, \Omega_{2}, \Omega_{3}\right)$ the angular (average spin) velocity of colloidal particles along z-axis, $I$ the moment of inertia, $\rho_{0}$ the reference density, $\mu_{0}$ the free space magnetic permeability, $\eta^{\prime}$ the shear spin viscosity co-efficient, $k_{1}$ the thermal conductivity, $T$ the temperature, $\alpha$ the thermal expansion co-efficient, $\delta$ the micropolar heat conduction coefficient, $C_{V, H}$ the specific heat at constant volume and magnetic field, $\boldsymbol{B}$ the magnetic induction field, $\boldsymbol{H}$ the magnetic field, $H$ the magnitude of $\boldsymbol{H}, H_{0}$ the constant applied magnetic field, $K=-(\partial M / \partial T)_{H_{0}, T_{0}}$ the pyromagnetic co-efficient, $\boldsymbol{M}$ the magnetization, $M$ the magnitude of $\boldsymbol{M}, M_{0}=M\left(H_{0}, T_{0}\right)$ the constant mean value of magnetization, $\chi=(\partial M / \partial H)_{H_{0}, T_{0}}$ the magnetic susceptibility, $\phi$ the magnetic potential and $\nabla^{2}=\partial^{2} / \partial x^{2}+\partial^{2} / \partial y^{2}+\partial^{2} / \partial z^{2}$ is the Laplacian operator.

The basic state is quiescent and is given by

$$
\begin{aligned}
p_{b}(z)= & p_{0}-\rho_{0} g z-\frac{\rho_{0} \alpha \beta g z^{2}}{2} \\
- & \frac{\mu_{0} M_{0} K \beta z}{(1+\chi)}-\frac{\mu_{0} K^{2} \beta^{2} z^{2}}{2(1+\chi)^{2}} \\
T_{b}(z)= & T_{0}-\beta z \\
\rho_{b}=\rho_{0} & {\left[1-\alpha\left(T_{b}-T_{0}\right)\right] } \\
\boldsymbol{H}_{b}(z)= & {\left[H_{0}-\frac{K \beta z}{(1+\chi)}\right] \hat{k} } \\
\boldsymbol{M}_{b}(z)= & {\left[M_{0}+\frac{K \beta z}{(1+\chi)}\right] \hat{k} }
\end{aligned}
$$

where, $\beta=\Delta T / d$ is the temperature gradient and the subscript $b$ denotes the basic state. 
To study the stability of the system, the variables are perturbed in the form

$$
\begin{aligned}
& \boldsymbol{q}=q^{\prime}, \boldsymbol{\omega}=\omega^{\prime}, p=p_{b}(z)+p^{\prime}, T=T_{b}(z)+T^{\prime}, \\
& \boldsymbol{H}=\boldsymbol{H}_{b}(z)+\boldsymbol{H}^{\prime}, \boldsymbol{M}=\boldsymbol{M}_{b}+\boldsymbol{M}^{\prime}
\end{aligned}
$$

where, $q^{\prime}, \omega^{\prime}, \rho^{\prime}, p^{\prime}, T^{\prime}, \boldsymbol{H}^{\prime}$ and $\boldsymbol{M}^{\prime}$ are the perturbed quantities whose magnitude is assumed to be very small.

Substituting Equation (11) in Equation (6a) and using Equations (8) and (9) and assuming $K \beta z \ll(1+\chi) H_{0}$ as propounded by Finlayson [6], we obtain (after dropping the primes)

$$
\begin{aligned}
& M_{x}+H_{x}=\left(1+\frac{M_{0}}{H_{0}}\right) H_{x}, M_{y}+H_{y}=\left(1+\frac{M_{0}}{H_{0}}\right) H_{y}, \\
& M_{z}+H_{z}=(1+\chi) H_{z}-K T
\end{aligned}
$$

where $\left(H_{x}, H_{y}, H_{z}\right)$ and $\left(M_{x}, M_{y}, M_{z}\right)$ are the $(x, y, z)$ components of the magnetic field and magnetization respectively.

Using Equation (11) in Equation (2) and linearizing, we obtain (after dropping primes)

$$
\begin{aligned}
\rho_{0} \frac{\partial u}{\partial t}= & -\left(\frac{\partial p}{\partial x}\right)+\left(\eta+\xi_{r}\right) \nabla^{2} u+2 \xi_{r} \Omega_{1} \\
& +\mu_{0}\left(M_{0}+H_{0}\right) \frac{\partial H_{1}}{\partial z} \\
\rho_{0} \frac{\partial v}{\partial t}= & -\left(\frac{\partial p}{\partial y}\right)+\left(\eta+\xi_{r}\right) \nabla^{2} v+2 \xi_{r} \Omega_{2} \\
& +\mu_{0}\left(M_{0}+H_{0}\right) \frac{\partial H_{2}}{\partial z} \\
\rho_{0} \frac{\partial w}{\partial t}= & -\left(\frac{\partial p}{\partial z}\right)+\left(\eta+\xi_{r}\right) \nabla^{2} w+2 \xi_{r} \Omega_{3} \\
& +\mu_{0}\left(M_{0}+H_{0}\right) \frac{\partial H_{3}}{\partial z}+\rho_{0} \alpha g T \\
& -\mu_{0} K \beta H_{3}+\frac{\mu_{0} \beta K^{2} T}{(1+\chi)} .
\end{aligned}
$$

Differentiating Equations (13)-(15) partially with respect to $x, y$ and $z$ respectively and adding, we get

$$
\begin{aligned}
\nabla^{2} p & =\mu_{0}\left(M_{0}+H_{0}\right) \nabla^{2}\left(\frac{\partial \phi}{\partial z}\right) \\
& +\left[\rho_{0} \alpha g+\frac{\mu_{0} \beta K^{2}}{(1+\chi)}\right] \frac{\partial T}{\partial z}-\mu_{0} \beta K\left(\frac{\partial^{2} \phi}{\partial z^{2}}\right)
\end{aligned}
$$

Eliminating the pressure term $p$ from Equation (15), using Equation (16) we get

$$
\begin{aligned}
& {\left[\rho_{0} \frac{\partial}{\partial t}-\left(\eta+\xi_{r}\right) \nabla^{2}\right] \nabla^{2} w=-\mu_{0} \beta K \nabla_{h}^{2}\left(\frac{\partial \phi}{\partial z}\right)} \\
& +\left[\rho_{0} \alpha g+\frac{\mu_{0} \beta K^{2}}{(1+\chi)}\right] \nabla_{h}^{2} T+2 \xi_{r} \nabla^{2} \Omega_{3}
\end{aligned}
$$

where, $\nabla_{h}^{2}=\partial^{2} / \partial x^{2}+\partial^{2} / \partial y^{2}$ is the horizontal Laplacian operator.

Substituting Equation (11) into Equation (3) and linearizing, we obtain (after dropping primes)

$$
\rho_{0} I \frac{\partial \Omega_{3}}{\partial t}=-2 \xi_{r}\left[\nabla^{2} w+2 \Omega_{3}\right]+\eta^{\prime} \nabla^{2} \Omega_{3} .
$$

As before, substituting Equation (11) into Equation (4) and linearizing, we obtain (after dropping primes)

$$
\begin{aligned}
{\left[\rho_{0} C_{0} \frac{\partial}{\partial t}-k_{1} \nabla^{2}\right] T } & =\left[\rho_{0} C_{0} \beta-\frac{\mu_{0} T_{0} \beta K^{2}}{(1+\chi)}\right] w \\
& +\mu_{0} T_{0} K \frac{\partial}{\partial t}\left(\frac{\partial \phi}{\partial z}\right)-\beta \delta \Omega_{3}
\end{aligned}
$$

where, $\rho_{0} C_{0}=\rho_{0} C_{V, H}+\mu_{0} K \boldsymbol{H}_{b}$. Finally Equations (6), after using Equation (12), yield (after dropping primes)

$$
(1+\chi) \frac{\partial^{2} \phi}{\partial z^{2}}+\left(1+\frac{M_{0}}{H_{0}}\right) \nabla_{h}^{2} \phi-K \frac{\partial T}{\partial z}=0 .
$$

The principle of exchange of stability is assumed and the normal mode expansion of the dependent variables is taken in the form

$$
\begin{aligned}
\left\{w, T, \phi, \Omega_{3}\right\}= & \left\{W(z), \Theta(z), \Phi(z), \Omega_{3}(z)\right\} \\
& \cdot \exp [\mathrm{i}(l x+m y)]
\end{aligned}
$$

where, $l$ and $m$ are the wave numbers in the $x$ and $y$ directions, respectively.

Let us non-dimensionalize the variables by setting

$$
\begin{gathered}
\left(x^{*}, y^{*}, z^{*}\right)=\left(\frac{x}{d}, \frac{y}{d}, \frac{z}{d}\right), D^{*}=D d, \quad a^{*}=a d, \\
W^{*}=\frac{d}{v} W, \Theta^{*}=\frac{\kappa}{\beta v d} \Theta, \\
\Phi^{*}=\frac{(1+\chi) \kappa}{K_{2} \beta v d^{2}} \Phi, \Omega_{3}^{*}=\frac{d^{3}}{v} \Omega_{3}, \quad I^{*}=\frac{1}{d^{2}} I
\end{gathered}
$$

where, $v=\eta / \rho_{0}$ is the kinematic viscosity and $\kappa=k_{1} / \rho_{0} C_{0}$ is the thermal diffusivity. Equation (21) is substituted into Equations (17)-(20) and then Equation (22) is used to obtain the stability equations in the following form:

$$
\begin{gathered}
\left(1+N_{1}\right)\left(D^{2}-a^{2}\right)^{2} W+a^{2} R_{t}\left[M_{1} D \Phi-\left(1+M_{1}\right) \Theta\right] \\
+2 N_{1}\left(D^{2}-a^{2}\right) \Omega_{3}=0 \\
2 N_{1}\left[\left(D^{2}-a^{2}\right) W+2 \Omega_{3}\right]-N_{3}\left(D^{2}-a^{2}\right) \Omega_{3}=0 \\
\left(D^{2}-a^{2}\right) \Theta+\left(1-M_{2}\right) W-N_{5} \Omega_{3}=0 \\
D^{2} \Phi-a^{2} M_{3} \Phi-D \Theta=0
\end{gathered}
$$

where $D=\mathrm{d} / \mathrm{d} z$ is the differential operator, 
$a=\sqrt{\ell^{2}+m^{2}}$ is the horizontal wave number,

$R_{t}=\alpha \beta g d^{4} / v \kappa$ is the thermal Rayleigh number,

$M_{1}=\mu_{0} K^{2} \beta /(1+\chi) \rho_{0} \alpha g$ is the magnetic number,

$M_{2}=\mu_{0} T_{0} K^{2} /(1+\chi) \rho_{0} C_{0}$ is the magnetic parameter,

$M_{3}=\left(1+M_{0} / H_{0}\right) /(1+\chi)$ is the non-linearity of mag-

netization, $N_{1}=\xi_{r} / \eta$ is the coupling parameter,

$N_{3}=\eta^{\prime} / \eta d^{2}$ is the spin diffusion (couple stress) parameter and $N_{5}=\delta / \rho_{0} C_{0} d^{2}$ is the micropolar heat conduction parameter. The typical value of $M_{2}$ for magnetic fluids with different carrier liquids turns out to be of the order of $10^{-6}$ and hence its effect is neglected when compared to unity.

Equations (23)-(26) are solved using the following boundary conditions:

i) Both boundaries rigid-isothermal and paramagnetic

$$
\begin{aligned}
& W=0=D W, \Omega_{3}=0, \Theta=0 \text { at } z=0,1 \\
& D \Phi= \begin{cases}a \Phi /(1+\chi), & \text { at } z=0 \\
-a \Phi /(1+\chi), & \text { at } z=1 .\end{cases}
\end{aligned}
$$

ii) Both boundaries rigid-isothermal and ferromagnetic

$$
W=0=D W, \Omega_{3}=0, \Theta=0, \Phi=0 \text { at } z=0,1 .
$$

\section{Numerical Solution}

Equations (23)-(26) together with the boundary conditions $(27 \mathrm{a}, \mathrm{b})$ or $(27 \mathrm{c})$ constitute an eigenvalue problem with the thermal Rayleigh number $R_{t}$ as the eigenvalue. For the boundary conditions considered, it is not possible to obtain the solution to the eigenvalue problem in closed form and hence it is solved numerically using the Galerkin-type weighted residuals method. Accordingly, the variables are written in a series of basis functions as

$$
\begin{aligned}
& W(z)=\sum_{i=1}^{N} A_{i} W_{i}(z), \Omega_{3}=\sum_{i=1}^{N} C_{i} \Omega_{3 i}(z), \\
& \Theta(z)=\sum_{i=1}^{N} D_{i} \Theta_{i}(z), \Phi(z)=\sum_{i=1}^{N} E_{i} \Phi_{i}(z)
\end{aligned}
$$

where, $A_{i}, C_{i}, D_{i}$ and $E_{i}$ are the unknown constants to be determined. The basis functions $W_{i}(z)$, $\Omega_{3 i}(z), \Theta_{i}(z)$ and $\Phi_{i}(z)$ are generally chosen such that they satisfy the corresponding boundary conditions but not the differential equations. Substituting Equation (28) into Equations (23)-(26), multiplying the resulting momentum equation by $W_{j}(z)$, angular momentum equation by $\Omega_{3 j}(z)$, temperature equation by $\Theta_{j}(z)$ and the magnetic potential equation by $\Phi_{j}(z)$; performing the integration by parts with respect to $z$ between $z=0$ and $z=1$ and using the boundary conditions $(27 \mathrm{a}, \mathrm{b})$ or (27c), we obtain the following system of $4 \mathrm{n}$ linear homogeneous algebraic equations in the $4 \mathrm{n}$ unknowns

$$
\begin{aligned}
A_{i}, C_{i}, D_{i} & \text { and } E_{i} ; i=1,2, \cdots, n: \\
& C_{j i} A_{i}+D_{j i} C_{i}+E_{j i} D_{i}+F_{j i} E_{i}=0 \\
& G_{j i} A_{i}+H_{j i} E_{i}=0 \\
& I_{j i} A_{i}+J_{j i} C_{i}+K_{j i} E_{i}=0 \\
& L_{j i} C_{i}+P_{j i} D_{i}=0 .
\end{aligned}
$$

The coefficients $C_{j i}-P_{j i}$ involve the inner products of the basis functions and are given by

$$
\begin{aligned}
& C_{j i}=\left(1+N_{1}\right)\left[\left\langle D^{2} W_{j} D^{2} W_{i}\right\rangle\right. \\
&\left.+2 a^{2}\left\langle D W_{j} D W_{i}\right\rangle+a^{4}\left\langle W_{j} W_{i}\right\rangle\right] \\
& D_{j i}=-a^{2} R_{t}\left(1+M_{1}\right)\left\langle W_{j} \Theta_{i}\right\rangle \\
& E_{j i}= a^{2} R_{t} M_{1}\left\langle W_{j} D \Phi_{i}\right\rangle \\
& F_{j i}=-2 N_{1}\left[\left\langle D W_{j} D \Omega_{3 i}\right\rangle+a^{2}\left\langle W_{j} \Omega_{3 i}\right\rangle\right] \\
& G_{j i}= 2 N_{1}\left[\left\langle D \Omega_{3 j} D W_{i}\right\rangle+a^{2}\left\langle\Omega_{3 j} W_{i}\right\rangle\right] \\
& H_{j i}=-\left[4 N_{1}\left\langle\Omega_{3 j} \Omega_{3 i}\right\rangle\right. \\
&\left.+N_{3}\left\{\left\langle D \Omega_{3 j} D \Omega_{3 i}\right\rangle+a^{2}\left\langle\Omega_{3 j} \Omega_{3 i}\right\rangle\right\}\right] \\
& I_{j i}=\left(1-M_{2}\right)\left\langle\Theta_{j} W_{i}\right\rangle \\
& J_{j i}=-\left[\left\langle D \Theta_{j} D \Theta_{i}\right\rangle+a^{2}\left\langle\Theta_{j} \Theta_{i}\right\rangle\right] \\
& K_{j i}=-N_{5}\left\langle\Theta_{j} \Omega_{3 i}\right\rangle \\
& L_{j i}=\left\langle\Phi_{j} D \Theta_{i}\right\rangle \\
& P_{j i}=\frac{a}{2(1+\chi)}+\left\langle D \Phi_{j} D \Phi_{i}\right\rangle+a^{2} M_{3}\left\langle\Phi_{j} \Phi_{i}\right\rangle
\end{aligned}
$$

where the inner product is defined as $\langle\cdot\rangle=\int_{0}^{1}(\cdot) \mathrm{d} z$.

The above set of homogeneous algebraic equations can have a non-trivial solution if and only if

$$
\left|\begin{array}{cccc}
C_{j i} & D_{j i} & E_{j i} & F_{j i} \\
G_{j i} & 0 & 0 & H_{j i} \\
I_{j i} & J_{j i} & 0 & K_{j i} \\
0 & L_{j i} & P_{j i} & 0
\end{array}\right|=0 .
$$

The eigenvalue has to be extracted from the above characteristic equation. For this, we select the trial functions as follows:

Case i): Rigid-paramagnetic boundaries

$$
\begin{aligned}
& W_{i}=\left(z^{4}-2 z^{3}+z^{2}\right) T_{i-1}^{*}, \Omega_{3 i}=\left(z^{2}-z\right) T_{i-1}^{*}, \\
& \Theta_{i}=\left(z^{2}-z\right) T_{i-1}^{*}, \Phi_{i}=(z-1 / 2) T_{i-1}^{*}
\end{aligned}
$$


Case ii): Rigid-ferromagnetic boundaries

$$
\begin{aligned}
& W_{i}=\left(z^{4}-2 z^{3}+z^{2}\right) T_{i-1}^{*}, \Omega_{3 i}=\left(z^{2}-z\right) T_{i-1}^{*}, \\
& \Theta_{i}=\left(z^{2}-z\right) T_{i-1}^{*}, \Phi_{i}=\left(z^{2}-z\right) T_{i-1}^{*}
\end{aligned}
$$

where $T_{i}^{*}{ }^{\prime} s$ are the modified Chebyshev polynomials. It may be noted that the trial function $\Phi_{i}$ does not satisfy the corresponding boundary conditions in the case of paramagnetic boundaries but the residual technique is used for the function $\Phi_{i}$ (see [6]) and the first term on the right hand side of $P_{j i}$ represents the residual term. In the case of ferromagnetic boundaries, $\Phi_{i}$ satisfies the corresponding boundary conditions and hence prevents the use of residual technique. Then the coefficient $P_{j i}$ is given by

$$
P_{j i}=\left\langle D \Phi_{j} D \Phi_{i}\right\rangle+a^{2} M_{3}\left\langle\Phi_{j} \Phi_{i}\right\rangle .
$$

The characteristic Equation (34) is solved numerically for different values of physical parameters using the Newton-Raphson method to obtain the Rayleigh number $R_{t}$ as a function of wave number $a$ and the bisection method is built-in to locate the critical stability parameters $\left(R_{t c}, a_{c}\right)$ to the desired degree of accuracy.

\section{Results and Discussion}

The classical linear stability analysis has been carried out to investigate the onset of ferromagnetic convection in a horizontal micropolar ferrofluid layer. The lower and upper boundaries are considered to be rigid-isothermal which are either paramagnetic or ferromagnetic. The critical thermal Rayleigh number $\left(R_{t c}\right)$ and the corresponding wave number $\left(a_{c}\right)$ are used to characterize the stability of the system. The critical stability parameters computed numerically by the Galerkin method as explained above, are found to converge by considering nine terms in the Galerkin expansion. To validate the numerical solution procedure used, a new magnetic parameter $S$, independent of the temperature gradient, was introduced in the form $R_{m}=R_{t}^{2} / S$, where $S=(1+\chi) \rho_{0} g^{2} \alpha^{2} d^{4} / \mu_{0} K^{2} \kappa v$. The critical thermal Rayleigh number $\left(R_{t c}\right)$, critical magnetic Rayleigh number $\left(R_{m c}\right)$ and the corresponding wave number $\left(a_{c}\right)$ computed numerically in the absence of micropolar effects $\left(N_{1}=N_{3}=N_{5}=0\right)$ are compared in Table 1 with the previously published results of Blennerhassett et al. [8]. It is seen that our results for different values of $S$ are in good agreement. Also, it is instructive to know the processes of convergence of results as the number of terms in the Galerkin approximation increases for the problem considered. Hence, various levels of the approximations to the critical thermal Rayleigh number $R_{t c}$ and the corresponding wave number are also obtained for different values of $N_{1}$ when $M_{3}=1, \quad R_{m}=R_{t} M_{1}=100$,
Table 1. Comparison of $\boldsymbol{R}_{t c}$ and $\boldsymbol{R}_{\boldsymbol{m}}$ for different values of $\boldsymbol{S}$ with $N_{1}=N_{3}=N_{5}=0$ (i.e., in the absence of micropolar effect). (a) When heated from below; (b) When heated from above.

(a)

\begin{tabular}{ccccccc}
\hline & \multicolumn{3}{c}{ Blennerhassett et al. [10] } & \multicolumn{3}{c}{ Present Analysis } \\
\cline { 2 - 7 } $\mathrm{S}$ & $R_{t c}$ & $a_{c}$ & $R_{m c}$ & $R_{t c}$ & $a_{c}$ & $R_{m c}$ \\
\hline 0 & 0 & 3.6088 & 2568.47 & 0 & 3.60874 & 2568.76 \\
$10^{-2}$ & 5.06 & 3.6075 & 2561.11 & 5.06102 & 3.60743 & 2561.39 \\
$10^{-1}$ & 15.95 & 3.6047 & 2545.24 & 15.9547 & 3.60462 & 2545.53 \\
1 & 49.96 & 3.5958 & 2495.69 & 49.9597 & 3.59579 & 2495.97 \\
10 & 153.13 & 3.5688 & 2344.99 & 153.142 & 3.56877 & 2345.26 \\
$10^{2}$ & 438.75 & 3.4920 & 1925.02 & 438.777 & 3.49195 & 1925.26 \\
$10^{3}$ & 1024.48 & 3.3252 & 1049.56 & 1024.55 & 3.32519 & 1049.71 \\
$10^{4}$ & 1552.74 & 3.1649 & 241.10 & 1552.88 & 3.16488 & 241.136 \\
$10^{5}$ & 1689.47 & 3.1221 & 28.54 & 1689.63 & 3.12208 & 28.5409 \\
$\infty$ & 1707.76 & 3.1163 & 0 & 1707.73 & 3.11638 & 0.0 \\
\hline
\end{tabular}

(b)

\begin{tabular}{ccccccc}
\hline & \multicolumn{3}{c}{ Blennerhassett et al. [10] } & \multicolumn{3}{c}{ Present Analysis } \\
\cline { 2 - 7 } $\mathrm{S}$ & $R_{t c}$ & $a_{c}$ & $R_{m c}$ & $R_{t c}$ & $a_{c}$ & $R_{m c}$ \\
\hline 0 & 0 & 3.6088 & 2568.47 & 0 & 3.60874 & 2568.76 \\
$10^{-2}$ & -5.08 & 3.6101 & 2575.9 & -5.08 & 3.61005 & 2576.15 \\
$10^{-1}$ & -16.10 & 3.6129 & 2591.9 & -16.10 & 3.61289 & 2592.19 \\
1 & -51.41 & 3.6220 & 2643.3 & -51.41 & 3.62197 & 2643.54 \\
10 & -167.69 & 3.6516 & 2811.9 & -167.69 & 3.65154 & 2812.22 \\
$10^{2}$ & -584.04 & 3.7536 & 3411.0 & -584.04 & 3.75355 & 3411.34 \\
$10^{3}$ & -2455.05 & 4.1464 & 6027.3 & -2455.05 & 4.1463 & 6027.65 \\
$10^{4}$ & -14797.1 & 5.5105 & 21895 & -14797.1 & 5.51039 & 21895.8 \\
$10^{5}$ & -119091 & 8.2382 & 141827 & -119091 & 8.23459 & 141816 \\
\hline
\end{tabular}

$N_{3}=2, N_{5}=1$ and the results are tabulated in Table 2 for different types of magnetic boundary conditions. It is seen that with an increase in the number of terms in the Galerkin approximation, $R_{t c}$ goes on decreasing and finally for the order $i=j=9$ the results converge. This clearly demonstrates the accuracy of the numerical procedure employed in solving the problem. The critical values obtained for different values of $N_{1}$ and $S$ as well as for two values of $N_{3}=2$ and 6 are exhibited in Table 3. It may be noted that as $S$ increases the magnetic Rayleigh number $R_{m}$ decreases, while the value of the critical Rayleigh number $R_{t c}$ increases. This implies that, in some favorable circumstances it is possible for the magnetic mechanism alone to induce convection.

The neutral stability curves ( $R_{t}$ against $a$ ) for different values of $M_{1}, N_{1}, N_{3}$ and $N_{5}$ are shown respectively in Figures 2-5 for paramagnetic/ferromagnetic boundaries. The neutral curves exhibit single but 
Table 2. Critical values of $R_{t c}$ and $a_{c}$ for different values of $N_{1}$ when $M_{3}=1, R_{m}=100, N_{3}=2$ and $N_{5}=1:$ (a) Paramagnetic boundaries when $1+\chi=8$; (b) Paramagnetic boundaries when $\chi=0$; (c) Ferromagnetic boundaries.

(a)

\begin{tabular}{cccccccccccc}
\hline \multirow{2}{*}{$N_{1}$} & \multicolumn{2}{c}{$i=j=1$} & \multicolumn{2}{c}{$i=j=3$} & \multicolumn{2}{c}{$i=j=5$} & \multicolumn{2}{c}{$i=j=8$} & \multicolumn{2}{c}{$i=j=9$} \\
\cline { 2 - 11 } & $R_{t c}$ & $a_{c}$ & $R_{t c}$ & $a_{c}$ & $R_{t c}$ & $a_{c}$ & $R_{t c}$ & $a_{c}$ & $R_{t c}$ & $a_{c}$ \\
\hline 0 & 1692.812 & 3.14012 & 1658.586 & 3.14784 & 1658.084 & 3.14792 & $\mathbf{1 6 5 8 . 0 8 3}$ & $\mathbf{3 . 1 4 7 9 2}$ & $\mathbf{1 6 5 8 . 0 8 3}$ & $\mathbf{3 . 1 4 7 9 2}$ \\
0.2 & 2529.982 & 3.11900 & 2495.969 & 3.13195 & 2495.065 & 3.13204 & $\mathbf{2 4 9 5 . 0 6 4}$ & $\mathbf{3 . 1 3 2 0 5}$ & $\mathbf{2 4 9 5 . 0 6 4}$ & $\mathbf{3 . 1 3 2 0 5}$ \\
0.4 & 3821.487 & 3.08148 & 3813.226 & 3.10318 & 3811.682 & 3.10335 & $\mathbf{3 8 1 1 . 6 8 1}$ & $\mathbf{3 . 1 0 3 3 6}$ & $\mathbf{3 8 1 1 . 6 8 1}$ & $\mathbf{3 . 1 0 3 3 6}$ \\
0.6 & 6049.422 & 3.00816 & 6166.736 & 3.04365 & 6164.059 & 3.04397 & $\mathbf{6 1 6 4 . 0 6 0}$ & $\mathbf{3 . 0 4 3 9 7}$ & $\mathbf{6 1 6 4 . 0 6 0}$ & $\mathbf{3 . 0 4 3 9 7}$ \\
0.8 & 10707.379 & 2.84549 & 11477.222 & 2.89729 & 11472.309 & 2.89794 & $\mathbf{1 1 4 7 2 . 3 1 7}$ & $\mathbf{2 . 8 9 7 9 4}$ & $\mathbf{1 1 4 7 2 . 3 1 7}$ & $\mathbf{2 . 8 9 7 9 4}$ \\
\hline
\end{tabular}

(b)

\begin{tabular}{cccccccccccc}
\hline & \multicolumn{2}{c}{$i=j=1$} & \multicolumn{2}{c}{$i=j=3$} & \multicolumn{2}{c}{$i=j=5$} & \multicolumn{2}{c}{$i=j=8$} & \multicolumn{2}{c}{$i=j=9$} \\
\cline { 2 - 11 } & $R_{t c}$ & $a_{c}$ & $R_{t c}$ & $a_{c}$ & $R_{t c}$ & $a_{c}$ & $R_{t c}$ & $a_{c}$ & $R_{t c}$ & $a_{c}$ \\
\hline 0 & 1673.024 & 3.12985 & 1644.147 & 3.13635 & 1643.612 & 3.13648 & $\mathbf{1 6 4 3 . 6 1 1}$ & $\mathbf{3 . 1 3 6 4 9}$ & $\mathbf{1 6 4 3 . 6 1 1}$ & $\mathbf{3 . 1 3 6 4 9}$ \\
0.2 & 2510.090 & 3.11210 & 2481.449 & 3.12425 & 2480.512 & 3.12438 & $\mathbf{2 4 8 0 . 5 1 1}$ & $\mathbf{3 . 1 2 4 3 9}$ & $\mathbf{2 4 8 0 . 5 1 1}$ & $\mathbf{3 . 1 2 4 3 9}$ \\
0.4 & 3801.401 & 3.07695 & 3798.544 & 3.09814 & 3796.967 & 3.09833 & $\mathbf{3 7 9 6 . 9 6 6}$ & $\mathbf{3 . 0 9 8 3 4}$ & $\mathbf{3 7 9 6 . 9 6 6}$ & $\mathbf{3 . 0 9 8 3 4}$ \\
0.6 & 6028.949 & 3.00539 & 6151.699 & 3.04056 & 6148.990 & 3.04090 & $\mathbf{6 1 4 8 . 9 9 1}$ & $\mathbf{3 . 0 4 0 9 0}$ & $\mathbf{6 1 4 8 . 9 9 1}$ & $\mathbf{3 . 0 4 0 9 0}$ \\
0.8 & 10686.048 & 2.84409 & 11461.278 & 2.89572 & 11456.336 & 2.89637 & $\mathbf{1 1 4 5 6 . 3 4 4}$ & $\mathbf{2 . 8 9 6 3 7}$ & $\mathbf{1 1 4 5 6 . 3 4 4}$ & $\mathbf{2 . 8 9 6 3 7}$ \\
\hline
\end{tabular}

(c)

\begin{tabular}{cccccccccccc}
\hline & \multicolumn{2}{c}{$i=j=1$} & \multicolumn{2}{c}{$i=j=3$} & \multicolumn{2}{c}{$i=j=5$} & \multicolumn{2}{c}{$i=j=8$} & \multicolumn{2}{c}{$i=j=9$} \\
\cline { 2 - 11 } & $R_{t c}$ & $a_{c}$ & \multicolumn{2}{c}{$R_{t c}$} & $a_{c}$ & $R_{t c}$ & $a_{c}$ & $R_{t c}$ & $a_{c}$ & $R_{t c}$ & $a_{c}$ \\
\hline 0 & 1649.975 & 3.11652 & 1628.295 & 3.12105 & 1627.728 & 3.12124 & $\mathbf{1 6 2 7 . 7 2 7}$ & $\mathbf{3 . 1 2 1 2 4}$ & $\mathbf{1 6 2 7 . 7 2 7}$ & $\mathbf{3 . 1 2 1 2 4}$ \\
0.2 & 2486.932 & 3.10307 & 2465.523 & 3.11397 & 2464.554 & 3.11414 & $\mathbf{2 4 6 4 . 5 5 3}$ & $\mathbf{3 . 1 1 4 1 5}$ & $\mathbf{2 4 6 4 . 5 5 3}$ & $\mathbf{3 . 1 1 4 1 5}$ \\
0.4 & 3778.005 & 3.07093 & 3782.424 & 3.09135 & 3780.815 & 3.09157 & $\mathbf{3 7 8 0 . 8 1 5}$ & $\mathbf{3 . 0 9 1 5 7}$ & $\mathbf{3 7 8 0 . 8 1 5}$ & $\mathbf{3 . 0 9 1 5 7}$ \\
0.6 & 6005.043 & 3.00158 & 6135.118 & 3.03633 & 6132.378 & 3.03668 & $\mathbf{6 1 3 2 . 3 8 0}$ & $\mathbf{3 . 0 3 6 6 8}$ & $\mathbf{6 1 3 2 . 3 8 0}$ & $\mathbf{3 . 0 3 6 6 8}$ \\
0.8 & 10660.918 & 2.84198 & 11443.449 & 2.89346 & 11438.477 & 2.89411 & $\mathbf{1 1 4 3 8 . 4 8 5}$ & $\mathbf{2 . 8 9 4 1 1}$ & $\mathbf{1 1 4 3 8 . 4 8 5}$ & $\mathbf{2 . 8 9 4 1 1}$ \\
\hline
\end{tabular}

Table 3. Critical values of $R_{t c}$ and $R_{m c}$ for different values of $N_{1}$ with $M_{3}=1$ and $N_{5}=1$.

\begin{tabular}{cccccccc}
\hline \multirow{2}{*}{$N_{3}$} & $N_{1}$ & \multicolumn{3}{c}{$S=10^{-2}$} & & \multicolumn{2}{c}{$S=10^{2}$} \\
\cline { 3 - 8 } & & $R_{t c}$ & $a_{c}$ & $R_{m c}$ & $R_{t c}$ & $a_{c}$ & $R_{m c}$ \\
\hline & 0 & 5.06079 & 3.60743 & 2561.15669 & 438.754 & 3.49195 & 1925.05399 \\
& 0.2 & 5.53993 & 3.60708 & 3069.08746 & 486.25 & 3.50052 & 2364.39091 \\
& 0.4 & 5.97264 & 3.60614 & 3567.24704 & 529.19448 & 3.50662 & 2800.46800 \\
& 0.6 & 6.36872 & 3.60496 & 4056.06846 & 568.53897 & 3.51117 & 3232.36568 \\
& 0.8 & 6.73495 & 3.60373 & 4535.95975 & 604.94257 & 3.51473 & 3659.55514 \\
& 0 & 5.06079 & 3.60743 & 2561.15669 & 438.754 & 3.49195 & 1925.05453 \\
& 0.2 & 5.54297 & 3.60738 & 3072.44943 & 486.554 & 3.50085 & 2367.34421 \\
& 0.4 & 5.98366 & 3.60709 & 3580.41825 & 530.297 & 3.50763 & 2812.15115 \\
& 0.6 & 6.39148 & 3.60665 & 4085.10372 & 570.816 & 3.51299 & 3258.31489 \\
& 0.8 & 6.77241 & 3.60614 & 4586.54893 & 608.691 & 3.51732 & 3705.04294 \\
\hline
\end{tabular}


different minimum with respect to the wave number and their shape is identical in the form to that of Benard problem in a micropolar fluid layer. For increasing $M_{1}$ (see Figure 2), $N_{1}$ (see Figure 3), $N_{5}$ (see Figure 4) and decreasing $N_{3}$ (see Figure 5), the neutral curves are slanted towards the higher wave number region. From the figures, it is also seen that increasing $\chi$ is to shift the neutral curves towards the higher wave number region. Moreover, the effect of increasing $M_{1}$ and $N_{3}$ as well as decreasing $N_{1}, N_{5}$ and $\chi$ is to decrease the region of stability.

Figure 6(a) represents the variation of critical Rayleigh number $R_{t c}$ as a function of $N_{1}$ for different values of $M_{1}$ and $\chi$ for $M_{3}=5, \quad N_{3}=2$ and

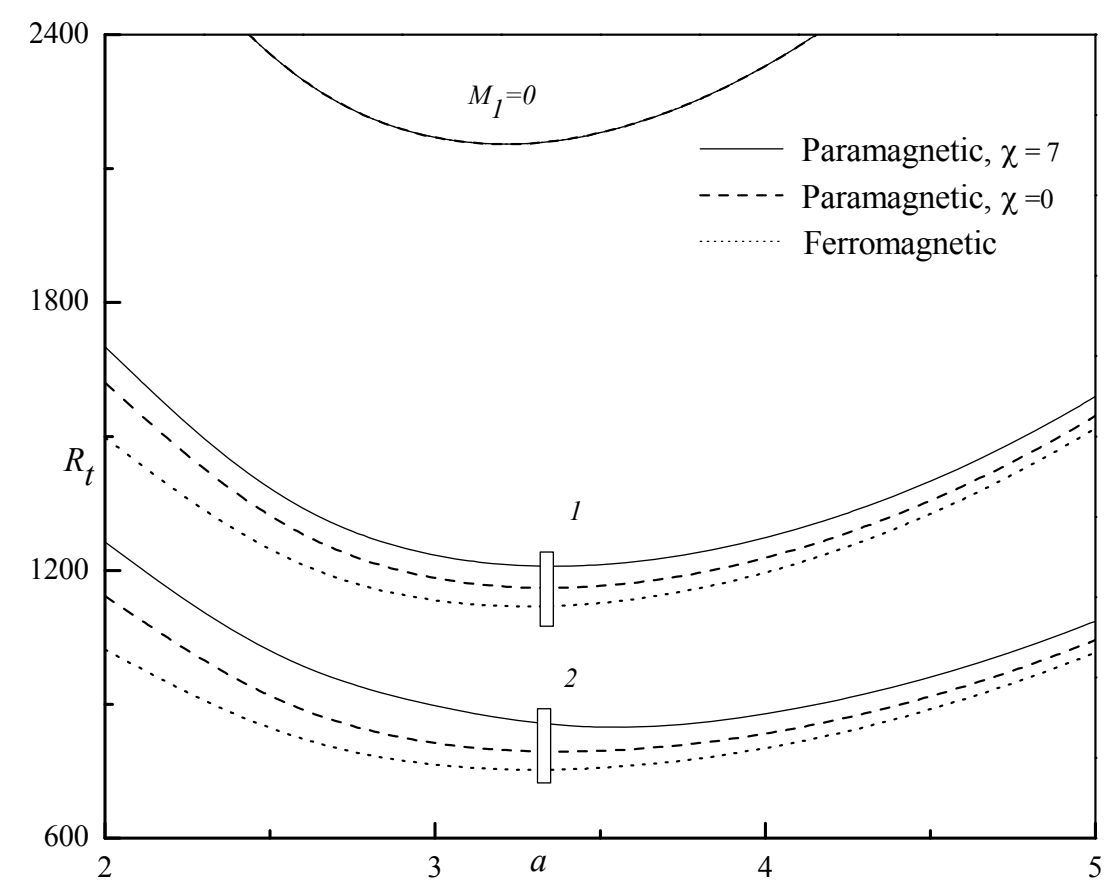

Figure 2. Neutral curves for different values of $M_{1}$ when $M_{3}=5, N_{1}=0.2, N_{3}=2$ and $N_{5}=0.5$.

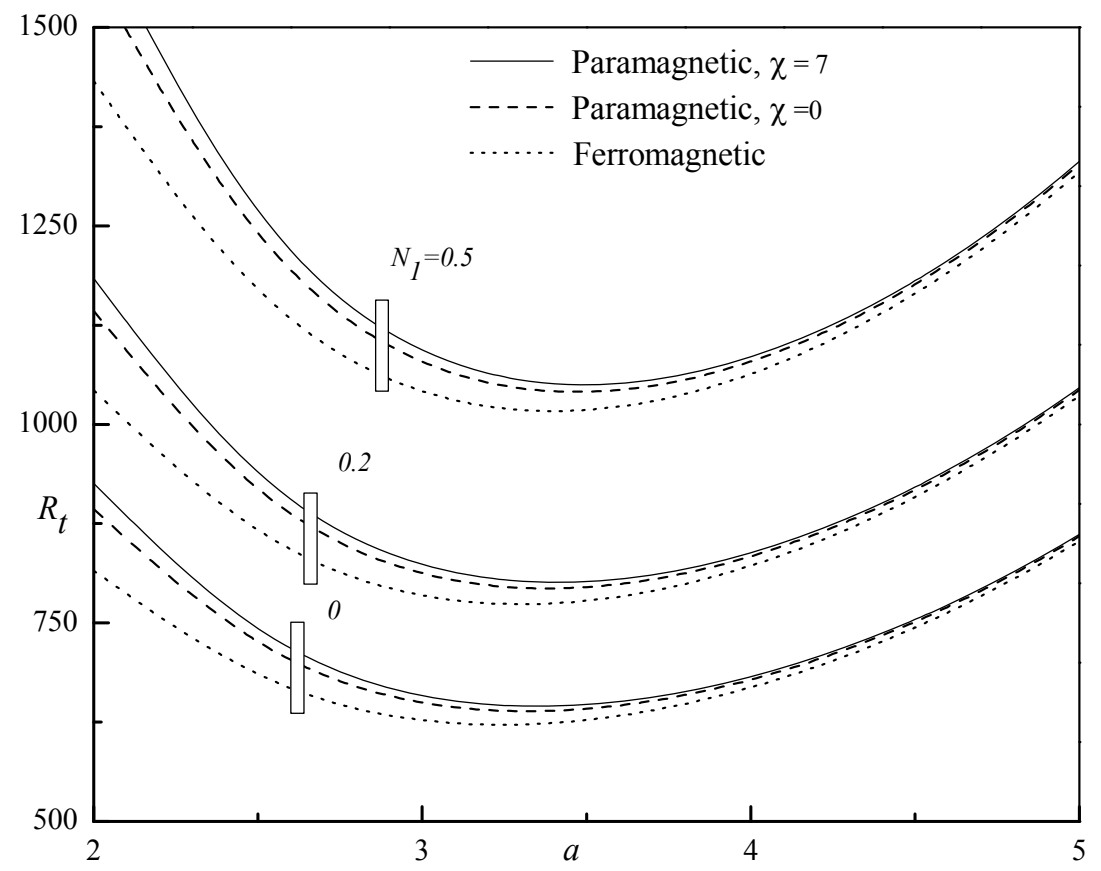

Figure 3. Neutral curves for different values of $N_{1}$ when $M_{1}=2, M_{3}=5, N_{3}=2$ and $N_{5}=0.5$. 


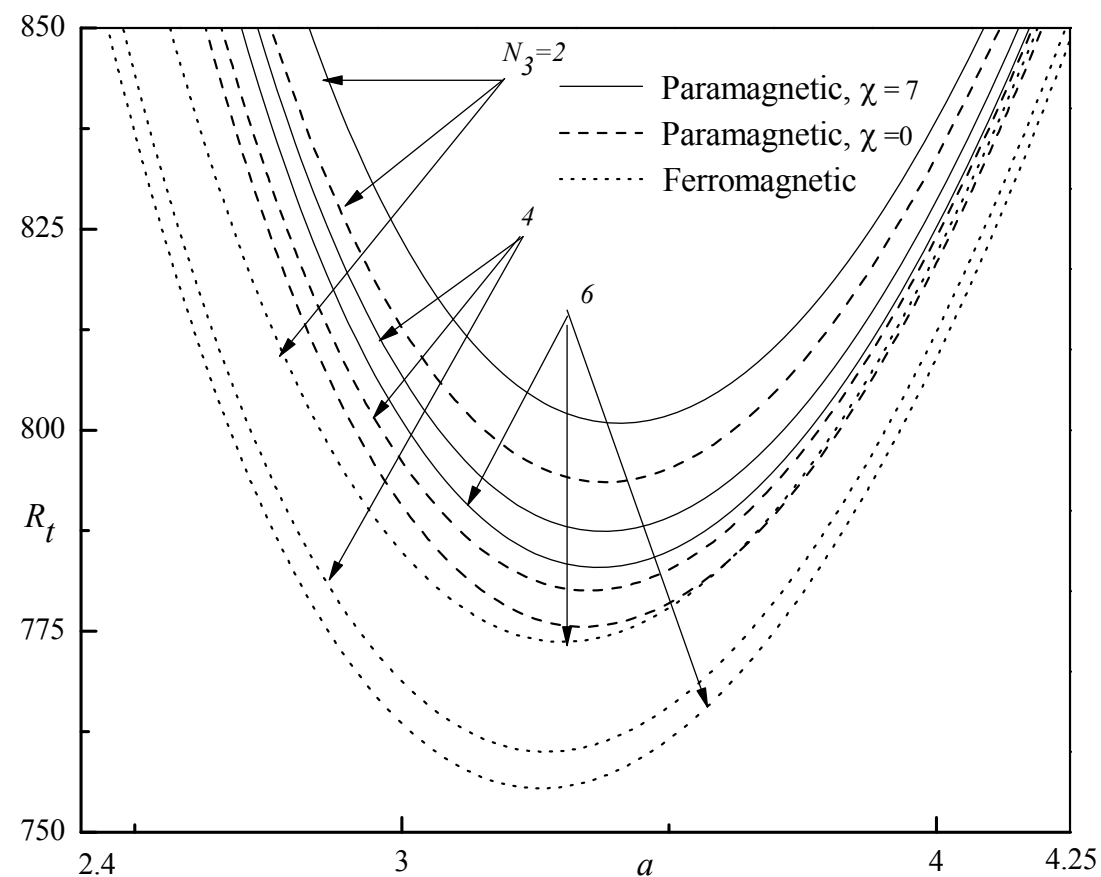

Figure 4. Neutral curves for different values of $N_{3}$ when $M_{1}=2, M_{3}=5, N_{1}=0.2$ and $N_{5}=0.5$.

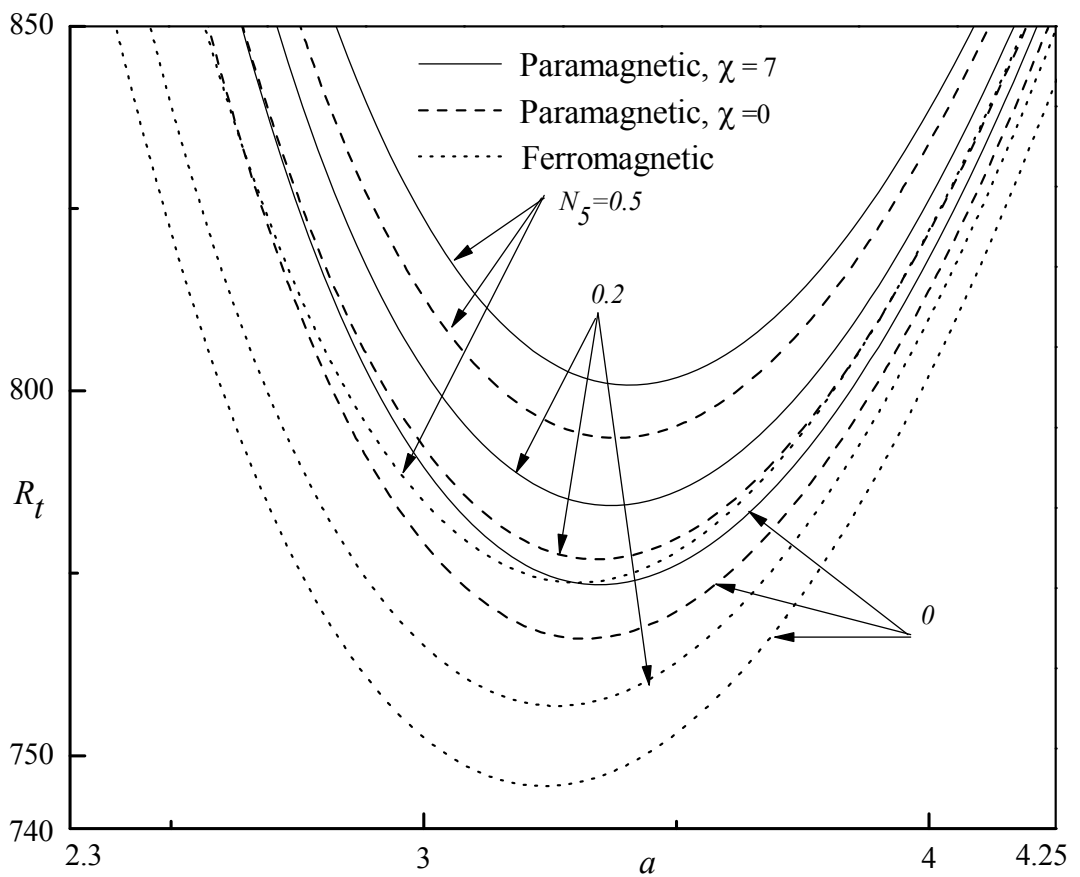

Figure 5. Neutral curves for different values of $N_{3}$ when $M_{1}=2, M_{3}=5, N_{1}=0.2$ and $N_{3}=2$.

$N_{5}=0.5$ for both ferromagnetic and paramagnetic boundary conditions. It is seen that $R_{t c}$ decreases with an increase in the value of $M_{1}$ and hence its effect is to hasten the onset of ferroconvection due to an increase in the destabilizing magnetic force and the curve for $M_{1}=0$ corresponds to non-magnetic micropolar fluid case. In other words, heat is transported more efficiently in magnetic fluids as compared to ordinary micropolar fluids. Also observed that $R_{t c}$ increases with increasing $N_{1}$. This is because, as $N_{1}$ increases the concentration of microelements also increases and as a result a greater part of the energy of the system is consumed by these elements in developing gyrational velocities in the fluid which ultimately leads to delay in the onset of ferromag- 
netic convection. Moreover, the system is found to be more stable if the boundaries are paramagnetic with $\chi=7$ as compared to the case of $\chi=0$ and the system is least stable if the boundaries are ferromagnetic. A closer inspection of the figure further depicts that the deviation in the $R_{t c}$ values for different magnetic boundary conditions is more pronounced with increasing coupling parameter. In Figure 6(b) plotted the critical wave number $a_{c}$ as a function of $N_{1}$. It is evident that increasing $N_{1}, \chi$ and $M_{1}$ is to increase the value of $a_{c}$ and thus their effect is to reduce the dimension of the convection cells.

In Figure 7(a) plotted $R_{t c}$ as a function of $N_{1}$ for different values of spin diffusion (couple stress) parameter $N_{3}$ when $M_{1}=2, M_{3}=5$ and $N_{5}=0.5$. Here, it is observed that $R_{t c}$ curves for different $N_{3}$ coalesce
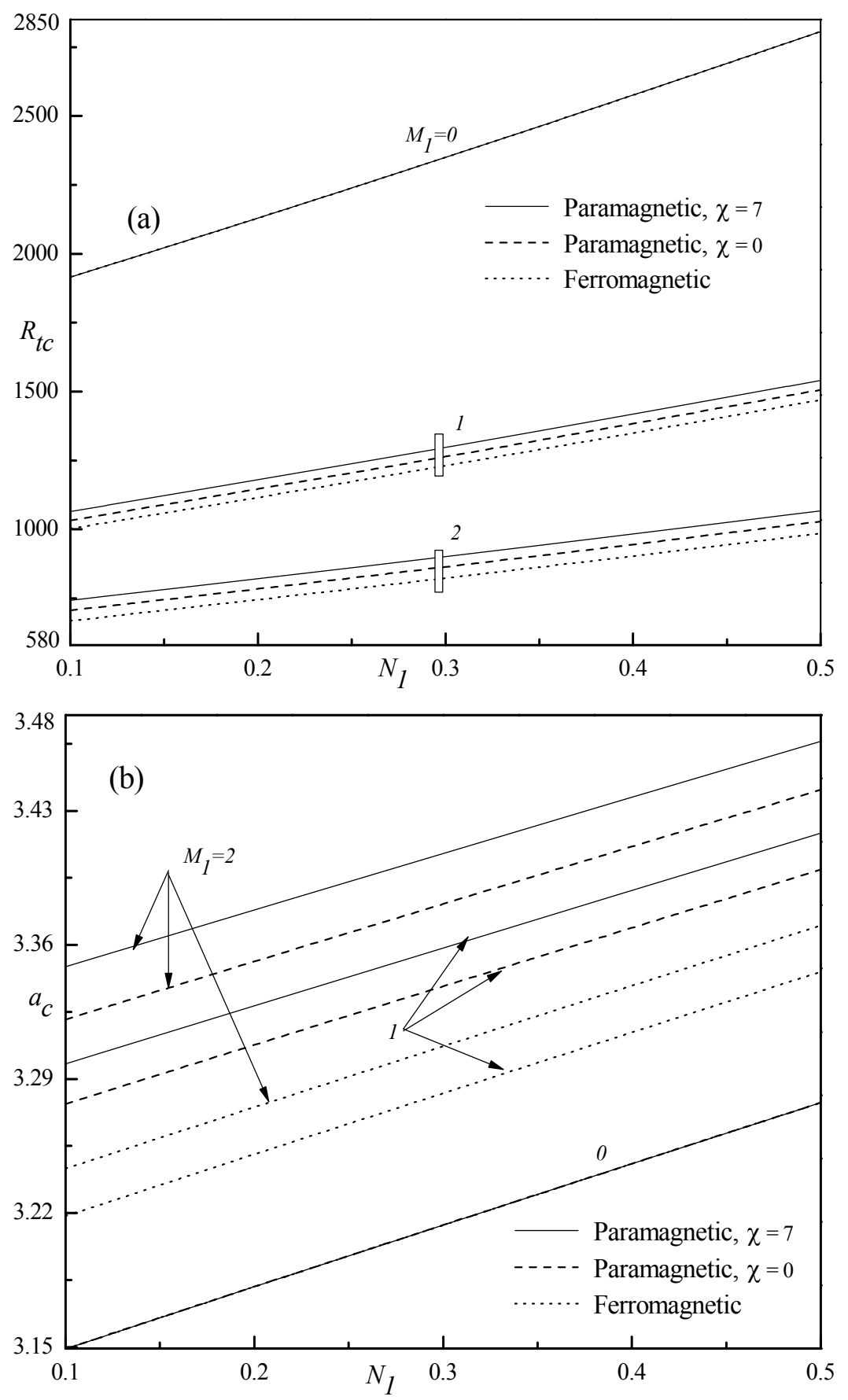

Figure 6. Variation of (a) $R_{t c}$ and (b) $a_{c}$ as a function of $N_{1}$ for different values of $M_{1}$ when $M_{3}=5, \quad N_{3}=2$ and $N_{5}=0.5$. 
when $N_{1}=0$. The impact of $N_{3}$ on the stability characteristics of the system is noticeable clearly with increasing $N_{1}$ and then it is seen that the critical Rayleigh number decreases with increasing $N_{3}$ indicating the spin diffusion (couple stress) parameter $N_{3}$ has a destabilizing effect on the system. This may be attributed to the fact that as $N_{3}$ increases, the couple stress of the fluid increases, which leads to a decrease in microrotation and hence the system becomes more unstable. Figure 7(b) illustrates that increase in $N_{1}$ and decrease in $N_{3}$ for non-zero values of $N_{1}$ is to increase $a_{c}$ and hence their effect is to decrease the size of convection cells.

The variation of critical thermal Rayleigh number $R_{t c}$ as a function of $N_{1}$ for different values of $N_{5}$ for $M_{1}=2, M_{3}=5$ and $N_{3}=2$ is shown in Figure 8(a). It is observed that increasing micropolar heat conduction parameter $N_{5}$ always has a stabilizing effect for nonzero values of $N_{1}$. When $N_{5}$ increases, the heat induced into microelements of the fluid is also increased, thus decreasing the heat transfer from the bottom to the top. This decrease in heat transfer is responsible for delaying the onset of ferromagnetic convection. Figure 8(b) illustrates that increase in $N_{1}$ and $N_{5}$ is to increase $a_{c}$ and hence their effect is to decrease the size of convection cells.

Figure 9 shows the locus of the critical thermal Rayleigh number $R_{t c}$ and the critical magnetic Rayleigh
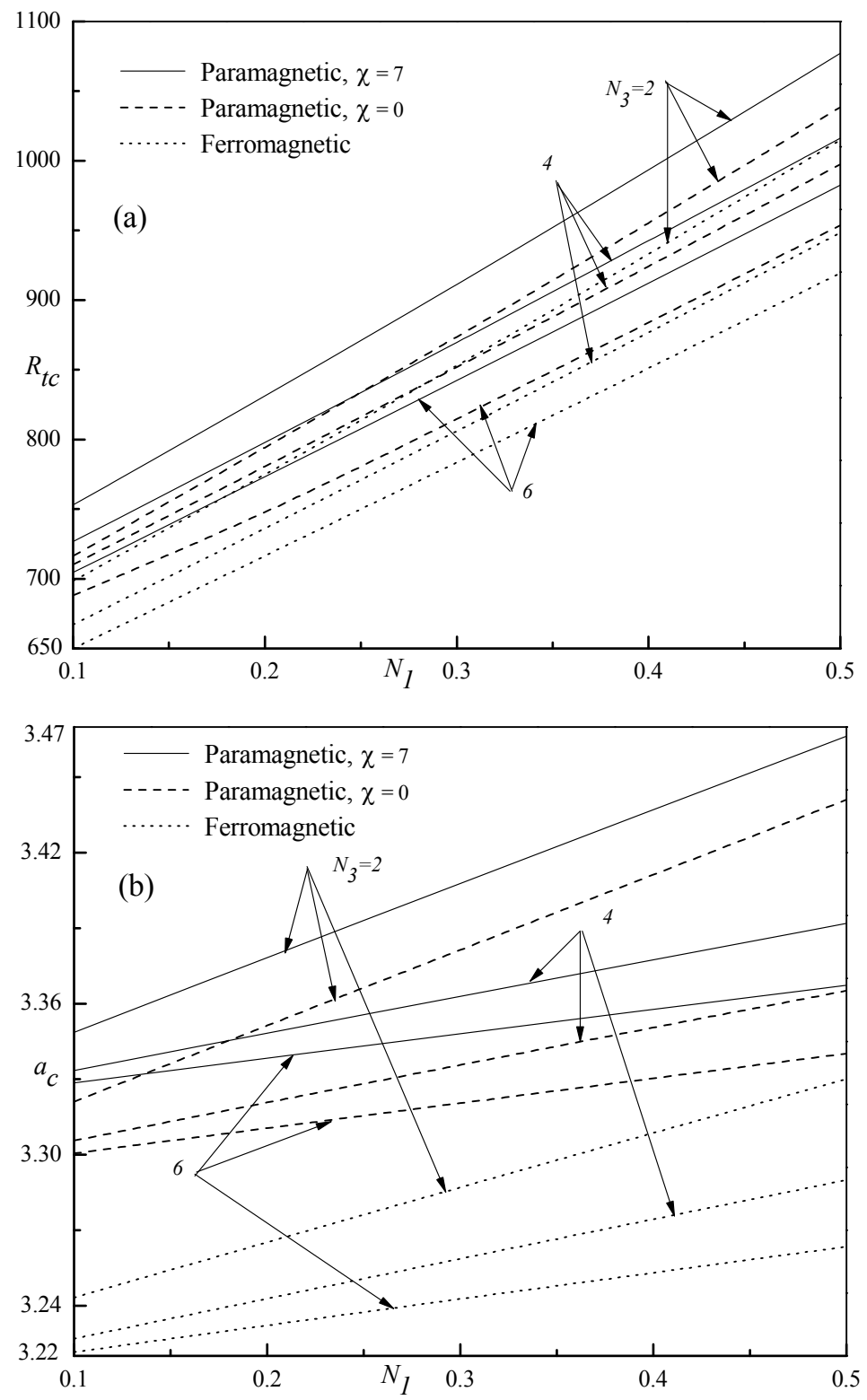

Figure 7. Variation of (a) $R_{t c}$ and (b) $a_{c}$ as a function of $N_{1}$ for different values of $N_{3}$ when $M_{1}=2, M_{3}=5$ and $N_{5}=0.5$. 

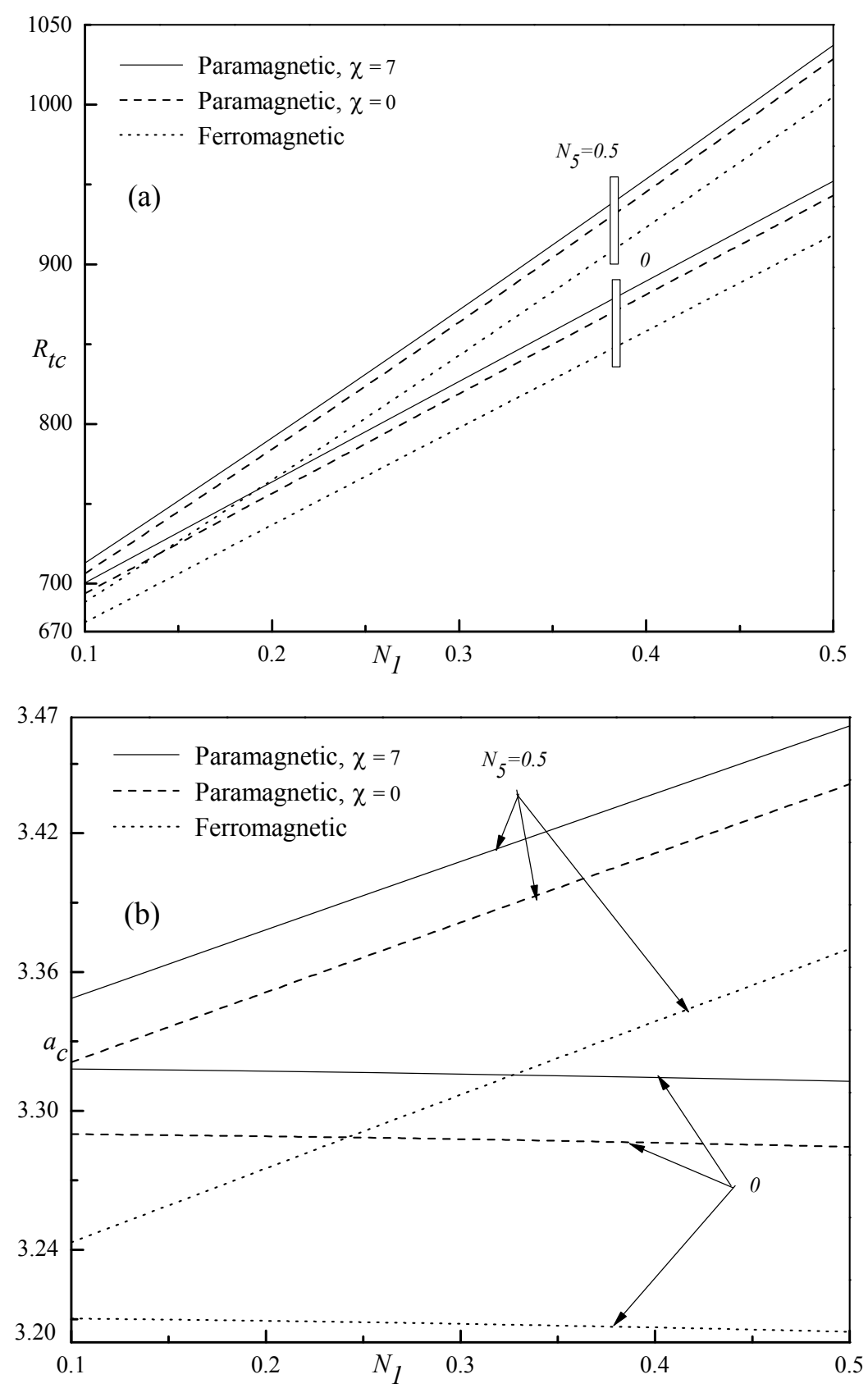

Figure 8. Variation of (a) $R_{t c}$ and (b) $a_{c}$ as a function of $N_{1}$ for different values of $N_{5}$ for $M_{1}=2, M_{3}=5$ and $N_{3}=2$.

$R_{m c}$ for $M_{3}=5, N_{1}=0.2, N_{3}=2$ and $N_{5}=0.5$. In the figure, the regions above and below the curves, correspond, respectively, to unstable and stable ones. It is observed that there is a strong coupling between the critical thermal Rayleigh and the magnetic Rayleigh numbers such that an increase in the one decreases the other. Thus, when the buoyancy force is predominant, the magnetic force becomes negligible and vice-versa. The stability curves are slightly convex and in the absence of buoyancy forces $\left(R_{t c}=0\right)$, the instability sets in at higher values of
$R_{m c}$ indicating the system is more stable when the magnetic forces alone are present. The stability region increases with increasing $\chi$ and also for paramagnetic boundaries when compared to ferromagnetic boundaries.

\section{Conclusions}

The linear stability theory is used to investigate the onset of ferromagnetic convection in a micropolar ferromagnetic fluid layer hated from below in the presence of a 


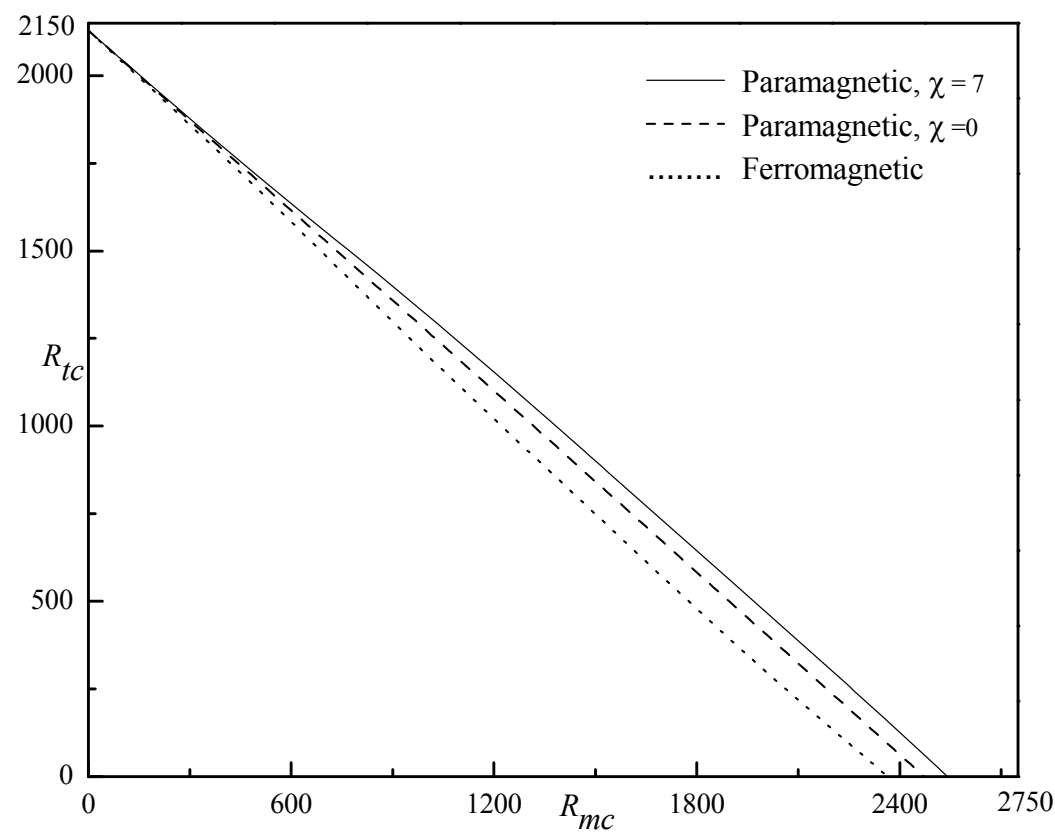

Figure 9. Locus of $R_{t c}$ and $R_{m c}$ for $M_{3}=5, \quad N_{1}=0.2, \quad N_{3}=2$ and $N_{5}=0.5$.

uniform applied vertical magnetic field for more realistic rigid boundary conditions which are considered to be either paramagnetic or ferromagnetic. The resulting eigenvalue problem is solved numerically by employing the Galerkin method.

From the foregoing study, the following conclusions may be drawn:

i) The neutral stability curves for various values of physical parameters exhibit that the onset of ferromagnetic convection retains its unimodal shape with one distinct minimum which defines the critical thermal Rayleigh number and the corresponding wave number.

ii) The system is more stabilizing against the ferromagnetic convection if the boundaries are paramagnetic with high magnetic susceptibility and least stable if the boundaries are ferromagnetic. It is observed that

$$
\begin{aligned}
\left(R_{t c} \text { and } a_{c}\right)_{\chi \neq 0} & >\left(R_{t c} \text { and } a_{c}\right)_{\chi=0} \\
& >\left(R_{t c} \text { and } a_{c}\right)_{\text {rigid-ferromagnetic }}
\end{aligned}
$$

iii) The effect of increasing the value of magnetic number $M_{1}$ is to hasten the onset of ferromagnetic convection.

iv) The effect of increasing the value of coupling parameter $N_{1}$ and micropolar heat conduction parameter $N_{5}$ is to delay, while increasing the spin diffusion (couple stress) parameter $N_{3}$ is to hasten the onset of ferromagnetic convection.

v) The effect of increasing $N_{1}, N_{5}, \chi$ and $M_{1}$ as well as decrease in $N_{3}$ is to increase the critical wave number.

vi) The magnetic and buoyancy forces are comple- mentary with each other and the system is more stabilizing when the magnetic forces alone are present.

\section{Acknowledgements}

The work reported in this paper was supported the Management (Panchajanya Vidya Peetha Welfare Trust) and Principal of Dr. Ambedkar Institute of Technology and East Point College of Engineering for Women, Bangalore for the encouragement.

\section{REFERENCES}

[1] R. E. Rosensweig, "Ferrohydrodynamics," Cambridge University Press, London, 1985.

[2] B. M. Berkovsky, V. F. Medvedev and M. S. Krakov, "Magnetic Fluids, Engineering Applications," Oxford University Press, Oxford, 1993.

[3] R. Hergt, W. Andrä, C. G. Ambly, I. Hilger, U. Richter and H. G. Schmidt, "Physical Limitations of Hypothermia Using Magnetite Fine Particles," IEEE Transictions of Magnetics, Vol. 34, No. 5, 1998, pp. 3745-3754. doi: $10.1109 / 20.718537$

[4] J. L. Neuringer and R. E. Rosensweig, "Magnetic Fluids," Physics of Fluids, Vol. 7, No. 12, 1964, pp. 1927-1937. doi:10.1063/1.1711103

[5] B. A. Finlayson, "Convective Instability of Ferromagnetic Fluids," Journal of Fluid Mechanics, Vol. 40, No. 4, 1970, pp. 753-767. doi:10.1017/S0022112070000423

[6] K. Gotoh and M. Yamada, "Thermal Convection in a Horizontal Layer of Magnetic Fluids," Journal of Physics, Society of Japan, Vol. 51, 1982, pp. 3042-3048. doi:10.1143/JPSJ.51.3042 
[7] P. J. Stiles, F. Lin and P. J. Blennerhassett, "Heat Transfer through Weakly Magnetized Ferrofluids," Journal of Colloidal and Interface Science, Vol. 151, No. 1, 1992, pp. 95-101. doi:10.1016/0021-9797(92)90240-M

[8] P. J. Blennerhassett, F. Lin and P. J. Stiles, "Heat Transfer through Strongly Magnetized Ferrofluids," Proceeding of Royal Society A: A Mathematical, Physical and Engineering Sciences, Vol. 433, 1991, pp. 165-177.

[9] Sunil and A. Mahjan, "A Nonlinear Stability Analysis for Magnetized Ferrofluid Heated from Below," Proceeding of Royal Society of London. A Mathematical, Physical and Engineering Sciences, Vol. 464, No. 2089, 2008, pp. 83-98. doi:10.1098/rspa.2007.1906

[10] C. E. Nanjundappa and I. S. Shivakumara, "Effect of Velocity and Temperature Boundary Conditions on Convective Instability in a Ferrofluid Layer," ASME Journal of Heat Transfer, Vol. 130, 2008, Article ID: 104502.

[11] M. I. Shliomis, "Convective Instability of Magnetized Ferrofluids: Influence of Magneto-Phoresis and Soret Effect," Thermal Non-Equilibrium Phenomena: Fluid Mixtures, Vol. 584, 2002, pp. 355-371.

[12] M. I. Shliomis and B. L. Smorodin, "Convective Instability of Magnetized Ferrofluids," Journal of Magnetism and Magnetic Materials, Vol. 252, 2002, pp. 197-202. doi:10.1016/S0304-8853(02)00712-6

[13] S. Odenbach, "Recent Progress in Magnetic Fluid Research," Journal of Physics: Condensed Matter, Vol. 16, 2004, pp. 1135-1150. doi:10.1088/0953-8984/16/32/R02

[14] P. J. Stiles and M. Kagan, "Thermoconvective Instability of a Ferrofluid in a Strong Magnetic Field," Journal Colloidal and Interface Science, Vol. 134, 1990, pp. 435448.

[15] P. N. Kaloni and J. X. Lou, "Convective Instability of Magnetic Fluids under Alternating Magnetic Fields," Physical Review E, Vol. 71, 2004, Article ID: 066311.

[16] P. Ram and K. Sharma, "Revolving Ferrofluid Flow under the Influence of MFD Viscosity and Porosity with Rotating Disk," Journal of Electromagnetic Analysis and Applications, Vol. 3, 2011, pp. 378-386. doi:10.4236/jemaa.2011.39060

[17] A. C. Eringen, "Simple Microfluids," International Journal of Engineering Sciences, Vol. 2, No. 2, 1964, pp. 205217. doi:10.1016/0020-7225(64)90005-9

[18] G. Lebon and C. Perez-Garcia, "Convective Instability of a Micropolar Fluid Layer by the Method of Energy," International Journal of Engineering Sciences, Vol. 19, 1981, pp. 1321-1329.

[19] L. E. Payne and B. Straughan, "Critical Rayleigh Numbers for Oscillatory and Non-Linear Convection in an Isotropic Thermomicropolar Fluid," International Journal of Engineering Sciences, Vol. 27, No. 7, 1989, pp. 827-836. doi:10.1016/0020-7225(89)90048-7

[20] P. G. Siddheshwar and S. Pranesh, "Effect of a Non-Uni- form Basic Temperature Gradient on Rayleigh-Benard Convection in a micropolar Fluid," International Journal of Engineering Sciences, Vol. 36, No. 11, 1998, pp. 11831196. doi:10.1016/S0020-7225(98)00015-9

[21] R. Idris, H. Othman and I. Hashim, "On Effect of NonUniform Basic Temperature Gradient on Bénard-Marangoni Convection in Micropolar Fluid," International Communications in Heat and Mass Transfer, Vol. 36, No. 3, 2009, pp. 255-258.

doi:10.1016/j.icheatmasstransfer.2008.11.009

[22] M. N. Mahmud, Z. Mustafa and I. Hashim, "Effects of Control on the Onset of Bénard-Marangoni Convection in a Micropolar Fluid," International Communications in Heat and Mass Transfer, Vol. 37, No. 9, 2010, pp. 13351339. doi:10.1016/j.icheatmasstransfer.2010.08.013

[23] S. Pranesh and R. V. Kiran, "Study of Rayleigh-Bénard Magneto Convection in a Micropolar Fluid with Maxwell-Cattaneo Law," Applied Mathematics, Vol. 1, 2010, pp. 470-480. doi:10.4236/am.2010.16062

[24] R. C. Sharma and P. Kumar, "On Micropolar Fluids Heated from Below in Hydromagnetics," Journal of Non-Equilibrium Thermodynamics, Vol. 20, No. 2, 1995, pp. 150159. doi:10.1515/jnet.1995.20.2.150

[25] R. C. Sharma and U. Gupta, "Thermal Convection in Micropolar Fluids in Porous Medium," International Journal of Engineering Sciences, Vol. 33, No. 11, 1995, pp. 1887-1892. doi:10.1016/0020-7225(95)00047-2

[26] M. Zahn and D. R. Greer, "Ferrohydrodynamics Pumping in Spatially Uniform Sinusoidally Time Varying Magnetic Fields," Journal of Magnetism and Magnetic Materials, Vol. 149, No. 1-2, 1995, pp. 165-173. doi:10.1016/0304-8853(95)00363-0

[27] A. Abraham, "Rayleigh-Benard Convection in a Micropolar Magnetic Fluids," International Journal of Engineering Sciences, Vol. 40, 2002, pp. 449-460.

[28] Reena and U. S. Rana, "Thermal Convection of Rotating Micropolar Fluid in Hydromagnetics Saturating a Porous Media," International Journal of Engineering. Transactions A: Basics, Vol. 21, No. 4, 2008, pp. 375-396.

[29] Reena and U. S. Rana, "Effect of Dust Particles on a Layer of Micropolar Ferromagnetic Fluid Heated from Below Saturating a Porous Medium," Applied Mathematics and Computation, Vol. 215, No. 7, 2009, pp. 2591-2607. doi:10.1016/j.amc.2009.08.063

[30] Y. Qin and P. N. Kaloni, “A Thermal Instability Problem in a Rotating Micropolar Fluid," International Journal of Engineering Sciences, Vol. 30, No. 9, 1992, pp. 11171126. doi:10.1016/0020-7225(92)90061-K

[31] Sunil, P. Chand, P. K. Bharti and A. Mahajan, "Thermal Convection a Micropolar Ferrofluid in the Presence of Rotation," Journal of Magnetism and Magnetic Materials, Vol. 320, No. 3-4, 2008, pp. 316-324. doi:10.1016/j.jmmm.2007.06.006 